\title{
Paradoxical response to osimertinib therapy in a patient with T790M-mutated lung adenocarcinoma
}

\author{
SHINICHIRO OKAUCHI ${ }^{1}$, HAJIME OSAWA ${ }^{1}$, KUNIHIKO MIYAZAKI ${ }^{2}$, MIO KAWAGUCHI $^{2}$ and HIROAKI SATOH ${ }^{1}$ \\ ${ }^{1}$ Division of Respiratory Medicine, Mito Medical Center, University of Tsukuba, Mito, Ibaraki 310-0015; \\ ${ }^{2}$ Division of Respiratory Medicine, Faculty of Medicine, University of Tsukuba, Tsukuba, Ibaraki 305-8575, Japan
}

Received April 11, 2017; Accepted August 17, 2017

DOI: $10.3892 / \mathrm{mco} .2017 .1474$

\begin{abstract}
A 'paradoxical response' to cancer treatment is a term used to describe the emergence of unexpected new lesions and the progression of existing lesions, despite appropriate and effective therapy. 'Pseudo-progression' is a phenomenon in which lymphocytes activated by an immune checkpoint inhibitor accumulate in a tumor and expand its shadow, mimicking enlargement of the primary lesion or development of a new metastatic lesion. Patients receiving cancer chemotherapy may respond differently to treatment, by exhibiting a response, deterioration, or the simultaneous occurrence of both. These variations may be attributed to the heterogeneity of the cancer. However, differences in the temporary response to epidermal growth factor receptor-tyrosine kinase inhibitor (EGFR-TKI) treatment are rarely observed. If such a phenomenon is observed, it should not affect the evaluation of the therapeutic effect or be considered as an indication for the discontinuation of treatment. We herein report a rare case of a transient increase in carcinomatous pleural fluid as a paradoxical response to osimertinib treatment in a patient with T790M-mutated lung adenocarcinoma. The primary lesion and pulmonary metastases responded well to therapy. Although this paradoxical response is very rare, of non-malignant nature, and does not usually require treatment modification of, physicians must acknowledge that it is not a clinically discouraging characteristic when using EGFR-TKI to treat T790M-mutated lung adenocarcinoma.
\end{abstract}

\section{Introduction}

A 'paradoxical response' to cancer treatment is the emergence of unexpected new lesions and/or the progression of existing lesions, despite appropriate and effective therapy. This term

Correspondence to: Professor Hiroaki Satoh, Division of Respiratory Medicine, Mito Medical Center, University of Tsukuba, Miya-machi 3-2-7, Mito, Ibaraki 310-0015, Japan

E-mail: hirosato@md.tsukuba.ac.jp

Key words: paradoxical response, osimertinib, epidermal growth factor receptor, T790M is commonly used in association with anti-tuberculosis treatment, where it describes a phenomenon observed in some patients with pulmonary and pleural tuberculosis, tuberculous lymphadenitis and intracranial tuberculoma (1-5). 'Pseudo-progression' is another term used to describe a phenomenon in which lymphocytes activated by an immune checkpoint inhibitor accumulate in a tumor to expand its shadow, appearing as enlargement of the primary lesion or development of a new metastatic lesion. A proportion of patients treated with nivolumab exhibit pseudo-progression, in which transient disease progression is followed by a response to an immune checkpoint inhibitor (6-10). Cancer patients may respond differently to chemotherapy by exhibiting a response, deterioration, or occasionally simultaneous occurrence of both. These different responses may be attributed to the heterogeneity of the cancer. However, differences in the transient responses to epidermal growth factor receptor-tyrosine kinase inhibitor (EGFR-TKI) treatment are rare and, should such a phenomenon be observed, it should not affect the evaluation of the therapeutic effect or be considered as an indication for discontinuation of treatment. We herein describe a case with a transient increase in the amount of carcinomatous pleural fluid as a paradoxical response to osimertinib therapy for T790M-mutated lung adenocarcinoma, despite the primary lesion and pulmonary metastases responding well to this treatment. The aim of the present study was to report this phenomenon in order to raise physicians' awareness of this rare occurrence.

\section{Case report}

A 58-year-old male patient was referred to the Mito Medical Center in January 2014 after experiencing dyspnea on exertion for 1 month. The patient had been diagnosed 27 months prior with adenocarcinoma in the upper lobe of the right lung. An EGFR exon 19 deletion was identified. Due to multiple metastases in both lungs, platinum-based chemotherapy and gefitinib therapy were administered. As third-line therapy, the patient received nivolumab therapy, but 3 months after the initiation of nivolumab therapy, a follow-up chest computed tomography (CT) scan revealed regrowth of the primary pulmonary lesion in the left upper lobe and appearance of miliary pulmonary metastases and bilateral pleural fluid (Fig. 1). The serum carcinoembryonic antigen (CEA) 

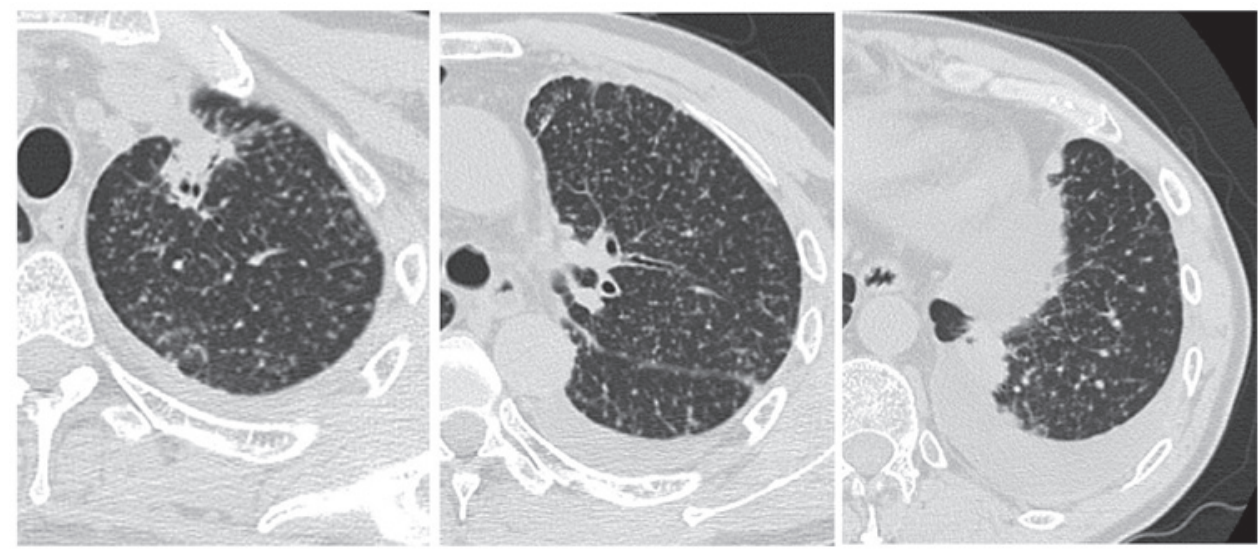

Figure 1. Chest computed tomography scan revealed regrowth of the primary lesion in the left upper lobe of the lung, with appearance of miliary pulmonary metastases and bilateral pleural fluid.

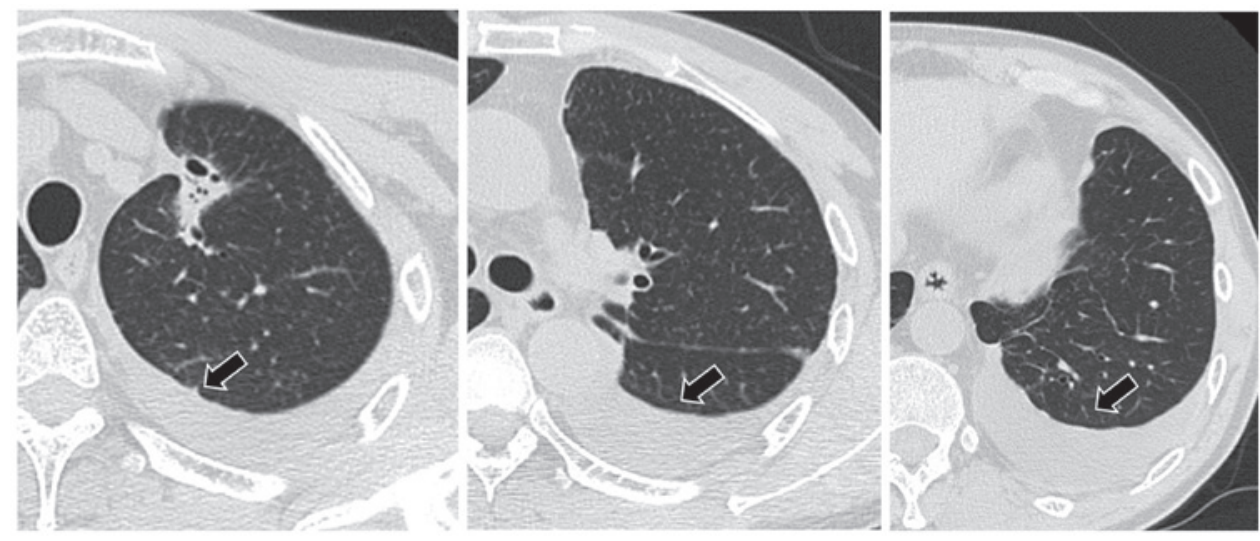

Figure 2. Chest computed tomography scan 1 month after the initiation of osimertinib treatment revealed shrinkage of the primary lesion and disappearance of pulmonary metastases, but an increase in the amount of pleural fluid was observed (arrows).
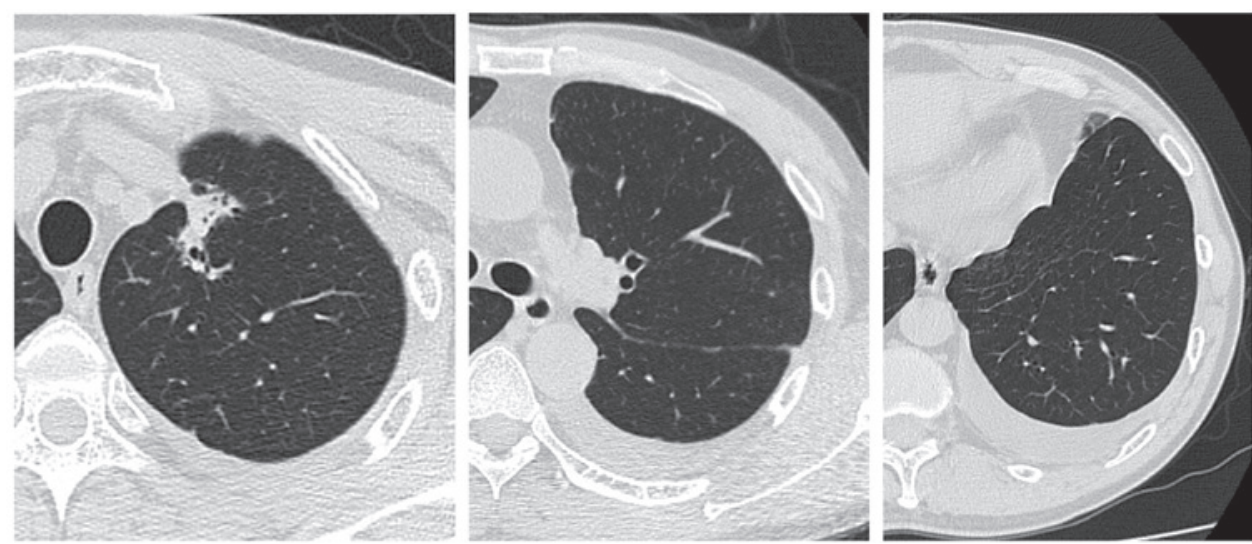

Figure 3. Chest computed tomography scan 1 month later showed decrease of the amount of pleural fluid and tumor shrinkage.

level increased from 252.4 to $340.2 \mathrm{ng} / \mathrm{ml}$ in 1 month. Thoracentesis was performed to obtain cytological specimens. Cytology of the pleural fluid revealed adenocarcinoma cells. A T790M mutation, together with a deletion within exon 19 of the EGFR gene, were identified. Osimertinib $(80 \mathrm{mg}$ once daily) was therefore prescribed as fourth-line treatment for the adenocarcinoma. A chest CT scan 1 month after the initiation of osimertinib treatment revealed shrinkage of the primary lesion and disappearance of pulmonary metastases, but an increase in the amount of pleural fluid (Fig. 2). A chest CT scan 1 month later revealed a decrease in the amount of the pleural fluid and tumor shrinkage (Fig. 3). In addition, the serum CEA level had decreased to $20.2 \mathrm{ng} / \mathrm{ml}$. The patient currently remains on osimertinib treatment, without major adverse events or disease recurrence (last follow-up visit, June 2017). 


\section{Discussion}

Pleural fluid is commonly found in a disseminated form in lung adenocarcinoma patients with carcinomatous pleuritis. However, to the best of our knowledge, an increase in the amount of pleural fluid as a transient paradoxical response to osimertinib therapy in a patient with EGFR-mutated lung cancer has never been reported to date. A paradoxical aggravation response, which is a phenomenon that appears despite effective chemotherapy for tuberculosis, is generally considered immune-mediated (1-5). Active tuberculosis leads to an immunosuppressed state via an altered cell-mediated response (1-5). When active tuberculosis is controlled by appropriate treatment, enhanced focal immune responses (immunological rebound) mobilize lymphocytes and macrophages at the site of lesions (2).

In a proportion of patients treated with nivolumab, an IgG4 antibody that targets the programmed cell death protein-1 on the T-cell surface, there is pseudo-progression followed by transient progression and then a response (6-8). This immune-related tumor response may result in a temporary increase in tumor size, regression, or emergence of new lesions, followed by a response to subsequent treatment (10). The response itself may take longer compared with that observed using conventional cytotoxic drugs (10). This pseudo-progression may be attributed to the inflammation, edema and necrosis that are associated with immune cell infiltration of the tumor deposits (9). This immunological response is important for characterizing the effects of immune checkpoint inhibitors, and is not observed with conventional cytotoxic drugs or other targeted therapeutics (8). The patient presented herein developed an increase in pleural fluid after 1 month of osimertinib therapy for recurrent T790M-mutated lung adenocarcinoma. The mechanism underlying this phenomenon has not been fully elucidated, but it may be hypothesized that rapid death of cancer cells following successful treatment of pulmonary and pleural lesions released factors that stimulated the production of pleural fluid. Another possibility is that increased pleural fluid is caused by an imbalance between production and drainage. It was unclear whether there was increased pleural fluid production, impaired drainage of pleural fluid, or both. There was no evidence to suggest pleurisy, other than carcinomatous pleuritis. The pleural fluid was exudative, but there were no findings consistent with infectious or tuberculous pleural fluid. It is paradoxical that there was a temporary increase in the pleural fluid containing T790M-positive cancer cells, despite the prompt response of the pulmonary metastatic lesions to treatment. Recognizing this clinically frustrating, but non-malignant paradoxical response, is meaningful for clinicians, although treatment modification is unnecessary. It is important that clinicians are aware of this rare event, whilst simultaneously considering other causes of an inadequate response to treatment, such as incorrect diagnosis, inappropriate drug regimen, drug resistance, atypical infectious disease, adverse effect to therapeutic drugs, or the deterioration of the disease. An accumulation of knowledge in this aspect of malignancy and cancer management will ensure continuing improvement in patient care. Informed consent to the publication of the case details and associated images was obtained from the patient.

\section{References}

1. Breen RA, Smith CJ, Bettinson H, Dart S, Bannister B, Johnson MA and Lipman MC: Paradoxical reactions during tuberculosis treatment in patients with and without HIV co-infection. Thorax 59: 704-707, 2004.

2. Marshall BG and Chambers MA: Central nervous system tuberculosis-the paradox of the host immune response. J Infect 36 : 3-4, 1998

3. Gupta RC, Dixit R, Purohit SD and Saxena A: Development of pleural effusion in patients during anti-tuberculosis therapy: Analysis of twenty-nine cases with review of literature. Indian J Chest Dis Allied Sci 42: 161-166, 2000.

4. Bukharie H: Paradoxical response to anti-tuberculosis drugs: Resolution with corticosteroid therapy. Scand J Infect Dis 32: 96-97, 2000.

5. Al-Orainey IO: Diagnosis of latent tuberculosis: Can we do better? Ann Thorac Med 4: 5-9, 2009.

6. Ito K, Hataji O, Katsuta K, Kobayashi T, Gabazza EC, Yatabe Y, Taguchi $\mathrm{O}$ and Yamamoto N: 'Pseudoprogression' of pulmonary pleomorphic carcinoma during nivolumab therapy. J Thorac Oncol 11: e117-e119, 2016.

7. Kolla BC and Patel MR: Recurrent pleural effusions and cardiac tamponade as possible manifestations of pseudoprogression associated with nivolumab therapy- a report of two cases. J Immunother Cancer 4: 80, 2016.

8. Tanizaki J, Hayashi H, Kimura M, Tanaka K, Takeda M, Shimizu S, Ito A and Nakagawa K: Report of two cases of pseudoprogression in patients with non-small cell lung cancer treated with nivolumab-including histological analysis of one case after tumor regression. Lung Cancer 102: 44-48, 2016.

9. Di Giacomo AM, Danielli R, Guidoboni M, Calabrò L, Carlucci D, Miracco C, Volterrani L, Mazzei MA, Biagioli M, Altomonte $\mathrm{M}$ and Maio M: Therapeutic efficacy of ipilimumab, an anti-CTLA-4 monoclonal antibody, in patients with metastatic melanoma unresponsive to prior systemic treatments: Clinical and immunological evidence from three patient cases. Cancer Immunol Immunother 58: 1297-1306, 2009.

10. Hoos A, Parmiani G, Hege K, Sznol M, Loibner H, Eggermont A, Urba W, Blumenstein B, Sacks N, Keilholz U, et al: A clinical development paradigm for cancer vaccines and related biologics. J Immunother 30: 1-15, 2007. 